\title{
Comparison of fluctuating maskers for speech recognition tests
}

Cite as:

Francart, T., Van Wieringen, A., Wouters, J. (2011). Comparison of fluctuating maskers for speech recognition tests. International Journal of Audiology, 50 (1), 2-13

Running title: Comparison of fluctuating maskers

Tom Francart ${ }^{1}$

ExpORL, Dept. Neurosciences, K.U.Leuven

Astrid van Wieringen ${ }^{2}$

ExpORL, Dept Neurosciences, K.U.Leuven

Jan Wouters ${ }^{3}$

ExpORL, Dept Neurosciences, K.U.Leuven

Corresponding author:

Tom Francart

ExpORL, Dept. Neurosciences, K.U.Leuven,

O \& N 2,

Herestraat 49 bus 721 ,

B-3000 Leuven, Belgium

tel: $+32(0) 16330476$

fax: +32 (0)16330486

tom.francart@med.kuleuven.be

\footnotetext{
${ }^{1}$ tom.francart@med.kuleuven.be

2 astrid.vanwieringen@med.kuleuven.be

33an.wouters@med.kuleuven.be
} 


\title{
List of acronyms
}

\author{
AM amplitude-modulated \\ ASA auditory scene analysis \\ $\mathbf{F}_{0} \quad$ fundamendal frequency \\ HI hearing impaired \\ IM informational masking \\ ISTS international speech test signal \\ LTASS long-term-average speech spectrum \\ NH normal hearing \\ SII speech intelligibility index \\ SNR signal-to-noise ratio \\ SRT speech recognition threshold \\ SWN speech-weighted noise \\ TFS temporal fine structure \\ TG temporal gap
}




\begin{abstract}
In speech recognition experiments, different maskers yield different performance at the same signal to noise ratio. The influence of properties such as temporal gaps, temporal fine structure and intelligibility on masking strength was investigated, for seven different masker types with Dutch speech materials, for normal-hearing and hearing-impaired subjects. Amongst the used maskers were the ICRA-5 fluctuating noise, the international speech test signal (ISTS), and competing talkers in Dutch and Swedish.

The normal-hearing subjects benefited from both temporal gaps and temporal fine structure in the fluctuating maskers. When the competing talker was comprehensible, performance decreased. The ISTS masker appeared to cause a large informational masking component. The stationary maskers yielded the steepest slopes of the psychometric function, followed by the modulated noises, followed by the competing talkers. Although the hearing-impaired group was heterogeneous, their data showed similar tendencies, but sometimes to a lesser extent, depending on individuals' hearing impairment.

If measurement time is of primary concern, non-modulated maskers are advised. If it is useful to assess release of masking by the use of temporal gaps, a fluctuating noise is advised. If perception of temporal fine structure is being investigated, a foreign-language competing talker is advised.
\end{abstract}




\section{Introduction}

Speech recognition tests are an important part of current clinical test batteries for hearing evaluation and are of great importance in research for assessing the performance of hearing aids and cochlear implants. To simulate realistic circumstances, speech tests are not only performed in silence, but also with an acoustic masker. In most cases a steady-state noise is used with the same long-termaverage spectrum as the speech material. However, depending on the objective of the test, certain types of maskers may yield more information.

Generally, masking can be divided into two different phenomena: energetic masking and informational masking (IM) (Durlach et al., 2003). Energetic masking is a mainly physical process where acoustic energy of the signal (at a certain time in a certain critical band) is masked by acoustic energy of the masker (present at the same time and in the same critical band). Informational masking, on the other hand, is related to the way the auditory system and more generally the brain process sounds. The separation of the incoming sound mixture into separate streams is called auditory scene analysis (ASA) (Bregman, 1990). Certain cues, such as pitch, temporal fine structure (TFS), voice timbre, onset/offset, spatial location, etc., are extracted from the incoming sound mixture. If the cues of the signal and masker differ, they can be used to segregate streams and if they are similar, they can have the opposite effect and confuse the listener, resulting in lower speech recognition performance. In the current study, spatial location cues are not considered. Temporal fine structure is defined as the rapid fluctuations in the signal, as opposed to the slowly changing envelope.

In the following paragraphs, different masker types and their effect on speech recognition will be described. We will discriminate between maskers based on three important properties: (1) the presence of temporal gaps (TGs), (2) the presence of TFS, and (3) whether the masker can be understood. For example, a stationary speech-weighted noise (SWN) has none of these properties, an amplitude-modulated (AM) SWN contains TGs, a competing talker in an unknown language contains both TGs and TFS and a competing talker in a language known to the subject has all three properties.

The presence of TGs in the masker can have a positive effect on speech recognition. Listeners can benefit from listening in the gaps in the masker, where the signal-to-noise ratio (SNR) is higher, and interpolate the remainder of the signal as to achieve higher performance than with a steady-state masker of the same overall intensity. This is called release of masking. Compared to normal-hearing $(\mathrm{NH})$ listeners, hearing-impaired (HI) listeners seem to be less able to benefit from TG in the masker, presumably because of reduced temporal and spectral resolution (Bacon et al., 1998; Eisenberg \& Dirks, 1995; Festen \& Plomp, 1990; Gustafsson \& Arlinger, 1994; Kwon \& Turner, 2001; Peters et al., 1998; Takahashi \& Bacon, 1992; Wagener et al., 2006). However, very recently Bernstein \& Grant (2009) indicated that the large differences between NH and HI subjects may at least partly be due to absolute differences in SNR used during the test; as HI subjects require more favorable SNRs for the same speech perception performance, higher SNRs are used during the test while the positive effect of TGs would become more salient at lower SNRs.

Festen \& Plomp (1990) found that the speech recognition threshold (SRT) ${ }^{1}$ of $20 \mathrm{NH}$ subjects improved by $4-6 \mathrm{~dB}$ when modulations were added to a steady-state noise, while HI subjects did not achieve a clear advantage of modulations in the masker. Gustafsson \& Arlinger (1994) assessed speech recognition performance with a steady-state masker compared to AM noises with various modulation parameters. The modulation frequency ranged from 2 to $100 \mathrm{~Hz}$ and the modulation depth from $100 \%$ to $6 \mathrm{~dB}$. Modulations were either sinusoidal or irregular. Normalhearing subjects obtained an advantage with both types of modulations. Release of masking was maximal for modulation frequencies between 10 and $20 \mathrm{~Hz}$ and for regular sinusoidal modulations.

${ }^{1}$ The SRT is the SNR at which a listener scores $50 \%$ correct. 
For HI subjects, release of masking was consistently smaller than for NH subjects. Peters et al. (1998) measured SRTs in noise with and without spectral and temporal dips. They found that NH subjects could use both types of dips to their advantage, while HI subjects were less able to take advantage of these. Wagener et al. (2006) assessed speech recognition performance with a steady-state masker and fluctuating noises with silent gaps of either 62.5 or $250 \mathrm{~ms}$. They found that both fluctuating noises differentiated well between HI subjects.

Above, only temporal gaps over the entire spectrum are considered. It is, however, also possible for temporal gaps to occur in only part of the spectrum. If the masker is divided in several frequency bands, one can think of this as comodulated versus uncomodulated gaps. Howard-Jones \& Rosen (1993) have shown that release of masking is possible due to glimpsing in an uncomodulated masker if the number of frequency bands is sufficiently small. While a competing talker can be considered uncomodulated, most gaps will be comodulated.

A more realistic masker than a modulated noise is a competing talker. However, IM can then start playing a role and its effect can vary depending on the similarity between the target speech and the masker and whether the competing talker can be understood by the subject. Shinn-Cunningham (2008) provides a framework to distinguish between two different types of IM. On the one hand, IM can occur due to failure in object formation. The auditory system forms objects (or streams) using acoustic properties of the target speech and the masker such as voice timbre, pitch, speech rate and SNR. If these properties are not properly perceived due to either energetic masking or hearing impairment, objects cannot be formed, leading to a reduction in speech perception performance. In what follows, this will be referred to as OFIM (IM due to a failure of object formation). On the other hand, if objects are properly formed, IM can still occur due to failure of object selection. In object selection, both top-down and bottom up processes play a role. To direct attention to a certain object (top-down), its signal must have properties that differentiate it from other objects and the listener needs to know which properties to use. If a competing object is more salient due to certain acoustic or perceptive properties, the top-down bias may be insufficient to override this bottom-up drawing of attention. This is also called distraction. Failure in object selection can lead to reduced speech recognition. In what follows we will indicate this as OSIM (IM due to failure in object selection).

When evaluating hearing aids and cochlear implants, it is important to assess whether the subject can perceive the acoustic properties (cues) needed for object formation. To avoid complicated OSIM, several techniques are used to prevent the subjects from understanding the masker, such as replacing the TFS by noise in the broadband signal, time reversing the masker, and using a competing talker in a foreign language. However, if the TFS is replaced by noise, the condition is similar to AM noise, which provides fewer object formation cues and is therefore less realistic. In addition, time reversing the competing talker can result in increased forward masking and therefore reduced speech recognition (Rhebergen et al., 2005).

Festen \& Plomp (1990) measured the SRT for $20 \mathrm{NH}$ and $20 \mathrm{HI}$ subjects and found for the NH listeners a $6-8 \mathrm{~dB}$ improvement in SRT for a competing talker over SWN. Rhebergen et al. (2005) tested recognition of Dutch sentences with a Dutch and a Swedish competing talker, both either unprocessed or time reversed. An estimated difference in SRT of $6.6 \mathrm{~dB}$ was attributed to IM due to the comprehensibility of the Dutch competing talker. Brungart (2001) tested speech perception performance with a competing talker as a function of SNR with same-talker, samesex and different-sex target and masker voices. The amount of masking was dependent on the similarity between target and masker voices. Performance did not always improve monotonically with increasing SNR. Sometimes the mere presence of intensity differences between target and masker improved performance with decreasing SNR. In other studies, the effect of multiple simultaneously competing talkers has been investigated. Generally, NH listeners benefit less from TGs and TFS with increasing number of talkers (Brungart et al., 2009, 2001; Cullington \& Zeng, 2008; Drullman \& Bronkhorst, 2004; Simpson \& Cooke, 2005). 
Currently, different maskers (noise materials) are used for the evaluation of hearing-aid signal processing systems. For instance, the international speech test signal (ISTS) was specifically developed for the technical evaluation of hearing aids (Holube, 2007), but it remains to be determined whether it is also suitable for use as a masker in speech recognition tests and how it compares to other types of commonly used maskers. Also, while the effects of the masker types mentioned above are described in the literature, they were tested with differing subject populations, differing speech materials and differing procedures and the secondary properties of the maskers used in different studies were not matched. It is therefore hard to compare the results between studies.

The current study assesses speech recognition performance of a group of NH and HI subjects with the three most used Dutch speech materials and seven different maskers. Reference values for $\mathrm{NH}$ subjects are given for the different conditions. The masking components attributable to energetic masking and IM are determined. Finally, suggestions are made concerning which maskers are best suited for clinical applications and research.

\section{Methods}

\section{$2.1 \quad$ Subjects}

Fifteen $\mathrm{NH}$ subjects and $10 \mathrm{HI}$ subjects were recruited. Of the NH subjects, eight were female and seven male. Their age at the time of testing varied between 18 and 22 years old. Their pure-tone hearing thresholds were better than $20 \mathrm{dBHL}$ at all octave audiometric frequencies from 250 to $8000 \mathrm{~Hz}$. If hearing thresholds differed between the two ears, the better ear was selected for the tests. If they did not differ, the right ear was tested (the left ear was tested in two of $15 \mathrm{NH}$ subjects).

Details for the HI subjects are given in Table I. Their ages ranged from 20 to 78 years old. All losses were purely sensorineural (maximal air-bone gap of $10 \mathrm{~dB}$ ) and largely symmetric. For each subject, the ear with the better thresholds was tested and no hearing aids were used during the tests. Audiograms of the tested ears are shown in Fig. 1.

\subsection{Materials}

\subsubsection{Speech materials}

The three most frequently used Dutch speech materials were used: the VU (male and female) and LIST (Leuven Intelligibility Sentence Test) sentence tests. The VU sentence test (Versfeld et al., 2000) consists of 39 lists of 13 sentences, uttered by a male and a female speaker (VUMan and VUWoman). A sentence is considered correct if all words, not only keywords, are repeated correctly.

The LIST sentence test (van Wieringen \& Wouters, 2008) consists of 35 lists of 10 sentences, uttered by a female speaker. Each sentence contains 3 or 4 keywords and each list contains 32 or 33 keywords. A sentence is considered correct if all keywords are repeated correctly and in the right order.

An overview of some speech material properties is given in Table II. 


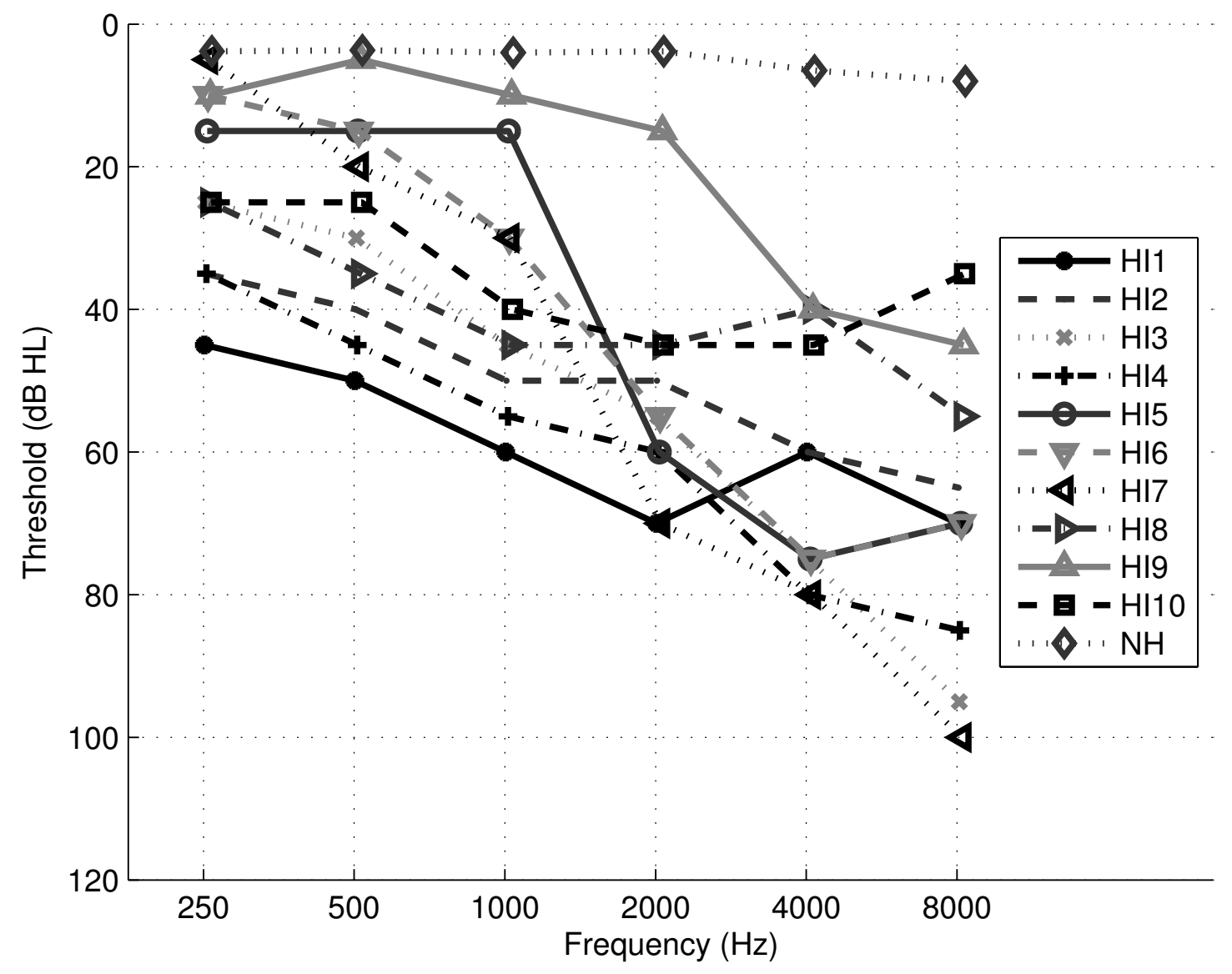

Figure 1: Average audiogram of the NH subjects and individual unaided audiograms for the HI subjects. 


\subsubsection{Maskers}

Seven different maskers were selected to vary in whether TGs were present, TFS information was present and, if so, whether the masker was comprehensible. Comparisons between pairs of maskers will elucidate the extent to which energetic and informational masking affect intelligibility for normal and impaired listeners.

The maskers used were the long-term-average speech spectrum (LTASS)-shaped noise corresponding to each speech material (SWN), a multitalker babble signal (MT), the ICRA5-250 noise, the kICRA noise (see below), a Swedish competing talker, a Dutch competing talker, and the ISTS signal. An overview of the different maskers and their properties is given in Table III. Extracts from the masker envelopes are shown in Fig. 2.

The multitalker babble signal (MT) was recorded at Auditec St-Louis and consists of a mixture of 20 English competing talkers (eight male, 12 female). The ICRA5-250 (International Collegium Of Rehabilitative Audiology) noise is described by Dreschler et al. (2001). In summary, it was developed by sending an English speech signal produced by a female talker through a three-channel filter bank, reversing the sign of each sample in each channel with a probability of 0.5 , filtering it again by the same filter bank, randomizing the phase in the frequency domain and applying the standard overall spectral shape of male speech (Byrne et al., 1994). The maximum silent gap length was limited to $250 \mathrm{~ms}$. The result is a male-spectrum-shaped wide-band noise that contains envelope modulations in three channels but no TFS.

The kICRA noise was created similarly to the ICRA5-250 noise, but now using the Dutch competingtalker recording (Cf. infra) as the input speech signal. The TG length was limited to $100 \mathrm{~ms}$ (in contrast to $250 \mathrm{~ms}$ in the ICRA5-250 noise). In this way, the kICRA has the same slow temporal structure of the Dutch competing talker and the same spectral properties of the ICRA5-250 noise.

For the Swedish and Dutch competing talkers, the story "The north wind and the sun" (IPA, 1999) was read in Swedish and Dutch by a native male Dutch speaker who was also fluent in Swedish. Multiple recordings were made of each sentence at different speech rates and the entire text was reassembled from recordings that did not contain any pronunciation errors and were very similar in speech rate and fundamendal frequency $\left(\mathrm{F}_{0}\right)$. Silent gaps longer than $100 \mathrm{~ms}$ were reduced to $100 \mathrm{~ms}$ using a custom Matlab program. Swedish was chosen because like Dutch it is a Germanic language, although the subjects could not understand it. The average $F_{0}$ and speech rate are shown in Table III.

The ISTS (International Speech Test Signal) was developed by the ISMADHA (International Standards for Measuring Advanced Digital Hearing Aids) working group of the European Hearing Instrument Manufacturing Association (EHIMA) for the purpose of analysing the processing of speech by hearing aids (Holube, 2007). It is a proposed standard signal that has all relevant properties of speech (i.e., the spectrum, modulations and fundamental frequency) and is not language-dependent and therefore internationally applicable. A native female speaker was selected for each of 6 different languages and the story "The north wind and the sun" (IPA, 1999) was recorded. The recordings were filtered to the standard LTASS of female speech (Byrne et al., 1994) and then split into segments of 100 to $600 \mathrm{~ms}$. The resulting segments were concatenated in random order with each language used once for each six sequential segments. The largest difference in $\mathrm{F}_{0}$ between two adjacent segments was $10 \mathrm{~Hz}$. We additionally processed the ISMADHA version of the ISTS signal to reduce the maximal silent gap length to $100 \mathrm{~ms}$.

The long term average spectra of the different materials were not matched to avoid modifying the standard materials and to allow comparison with previously published data. We did, however, calculate the speech intelligibility index (SII) according to ANSI-3.5 1997, using the band-importance 

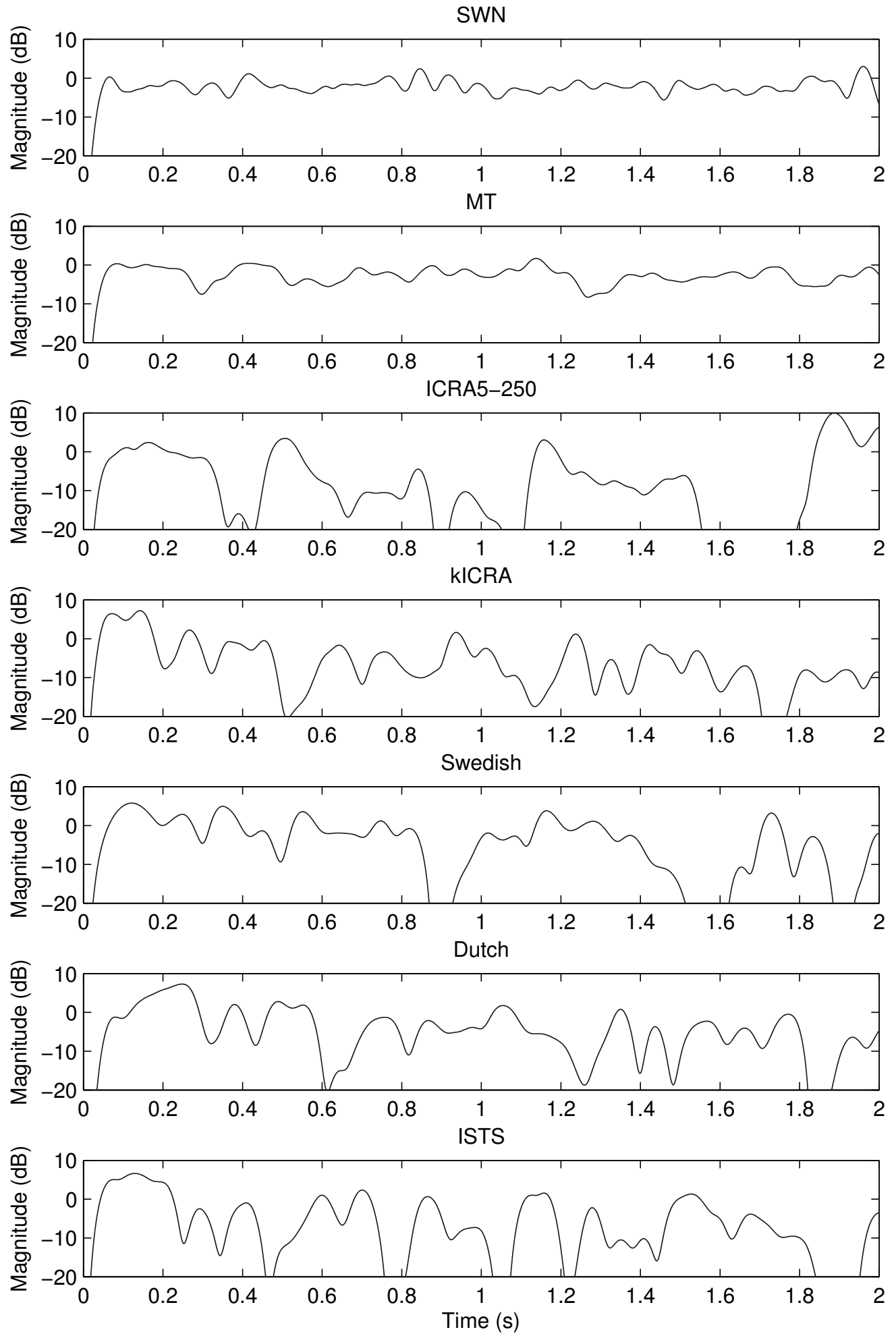

Figure 2: Extract of the masker envelopes, determined by full wave rectification and low pass filtering $\left(\mathrm{f}_{\mathrm{c}}=25 \mathrm{~Hz}\right)$ of an extract of the masker. All input signals were normalized to the same RMS level. 


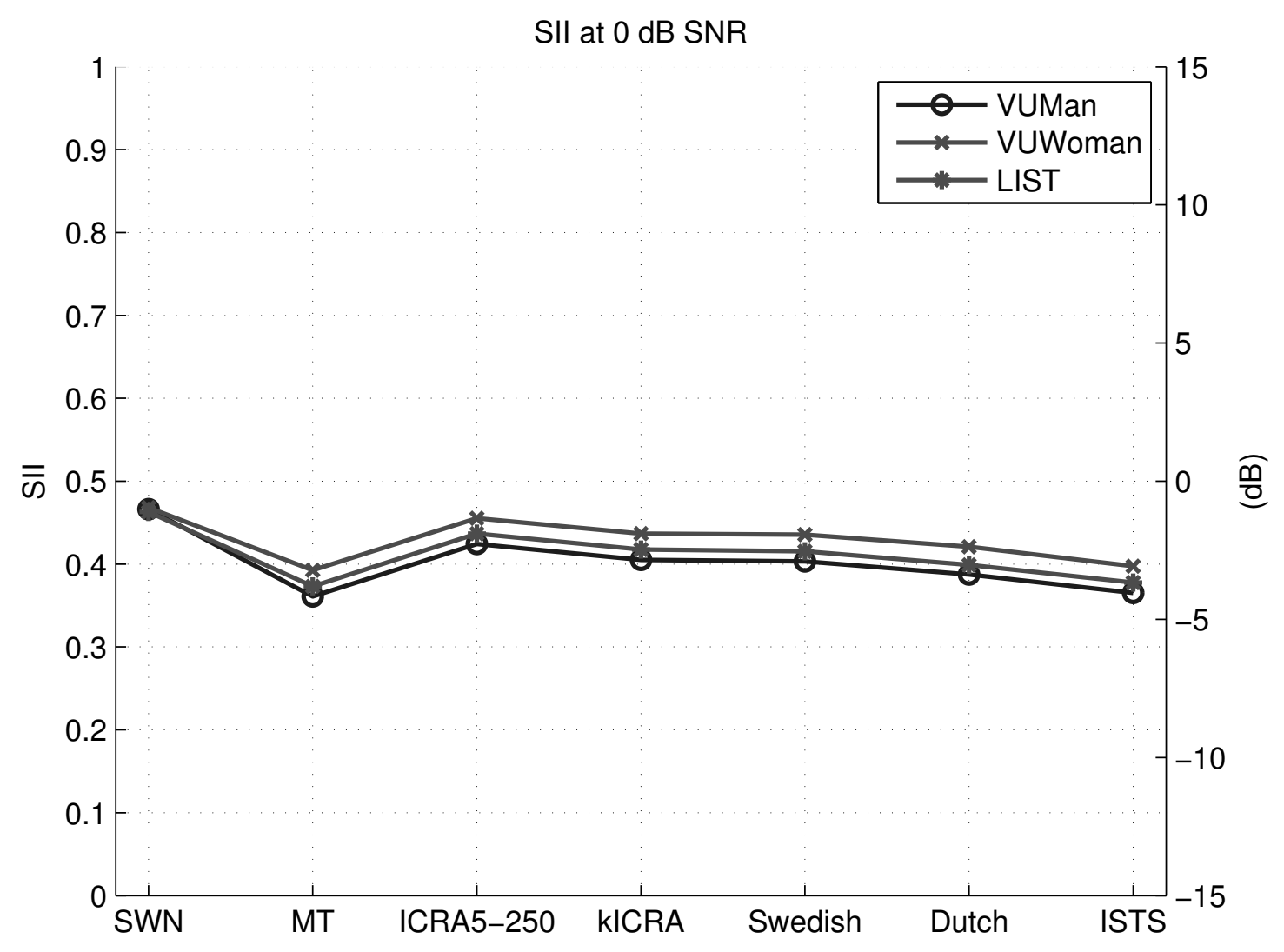

Figure 3: SII at $0 \mathrm{~dB}$ SNR for each combination of speech material and masker. The axis on the right hand side indicates that a difference in SII of 0.1 corresponds to a difference in SRT of approximately $3 \mathrm{~dB}$.

function for speech in noise (table B.2), to determine the spectral influence for each combination of speech material and masker. The results for an SNR of $0 \mathrm{~dB}$ are shown in Fig. 3. A difference in SII of 0.1 corresponds to a difference in SRT of approximately $3 \mathrm{~dB}$ (ANSI-3.5 1997), as indicated by the axis on the right hand side of Fig. 3. These differences will be taken into account in the discussion.

\subsection{Apparatus}

The tests took place in a silent room or a sound booth. Speech and noise were presented monaurally to the subject using the APEX 3 program, developed at ExpORL (Francart et al., 2008). An RME Hammerfall DSP soundcard and Sennheiser HD250 Linear II headphones were used. Each masker was set to $60 \mathrm{dBA}$ using the B\&K artificial ear 4153 and speech was added according to the desired SNR, which was calculated using the RMS energy of the signals as stored on disk.

\subsection{Procedure}

The SRT was determined using an adaptive procedure (Plomp \& Mimpen, 1979). A run consisted of a list of ten (LIST) or thirteen (VU) sentences. The masker was presented continuously during 
an adaptive run. The level of the masker was held constant at $60 \mathrm{dBA}$. The first sentence was presented at an SNR sufficiently low such that it was not recognized. Then the SNR was increased in steps of $2 \mathrm{~dB}$ until the sentence was recognized. Subsequently each successive sentence was presented once and the SNR was varied in steps of $2 \mathrm{~dB}$ in a 1-up 1-down procedure, targeting the $50 \%$ correct point. After determining the level of the imaginary last trial, the SRT was calculated as the average of the last 6 SNRs, including the imaginary last trial.

A condition consisted of a combination of a speech material and masker. For each subject, the SRT was determined for each possible condition, both as test and retest on different days. This resulted in a total of 3 (speech materials) $\times 7$ (maskers) $\times 2$ (repetitions) $=42$ lists of sentences administered to each subject. The order of tests was randomized using a Latin square and care was taken to only present a sentence list once to each subject to avoid learning effects.

\section{Results}

\subsection{Slopes and variability}

The slope of the psychometric function for an SRT test indicates the precision of the test when using a limited number of trials (limited testing time). As the slope cannot be directly derived from the staircase of the adaptive procedure, the data points from the adaptive runs of the different conditions were converted to performance-intensity points and a psychometric function with parameters slope and SRT was fitted to these points for each condition, using a non-linear least-squares method. The data points of the first sentence were not included in the fit. The results for the different $\mathrm{NH}$ subjects were merged to obtain a sufficient number of data points per condition. Note that combining data points for the different subjects can result in shallower slopes if there are large inter-subject differences in 50\% point (i.e., in SRT). The slopes are shown in Fig. 4. The error bars indicate 95\% confidence intervals of the fit. If the error bars do not overlap, two conditions are considered significantly different. In all cases except for Dutch/LIST and Dutch/VUMan, there were no significant differences between speech materials for the same masker. Wilcoxon Rank-sum tests indicated significant differences $(p<0.01)$ between the slopes of the steady-state maskers (MT and SWN) and the modulated noises (ICRA5-250 and kICRA) and between the modulated noises and the competing talkers (Swedish, Dutch and ISTS). The slopes were steepest for the steady-state maskers (MT and SWN). The slopes were shallowest for the competing talkers (Swedish, Dutch and ISTS). Note that the speech materials were designed to allow efficient adaptive threshold determination with the accompanying SWN. During the development of the LIST and VU speech materials, sentences were selected that yielded similar SRTs in the corresponding LTASS weighted noises. If a fluctuating noise would be used during the development, a steeper slope for fluctuating noise might result.

In Fig. 5 the mean absolute differences between test and retest are shown for the NH subjects. These indicate the test reproducibility. Note that as the absolute value of the difference is used, they do not indicate better performance during the retest. Based on the error bars, there are no significant differences between conditions, but similar to the slopes, there is a tendency of larger differences for the fluctuating maskers and even larger for maskers containing TFS. 


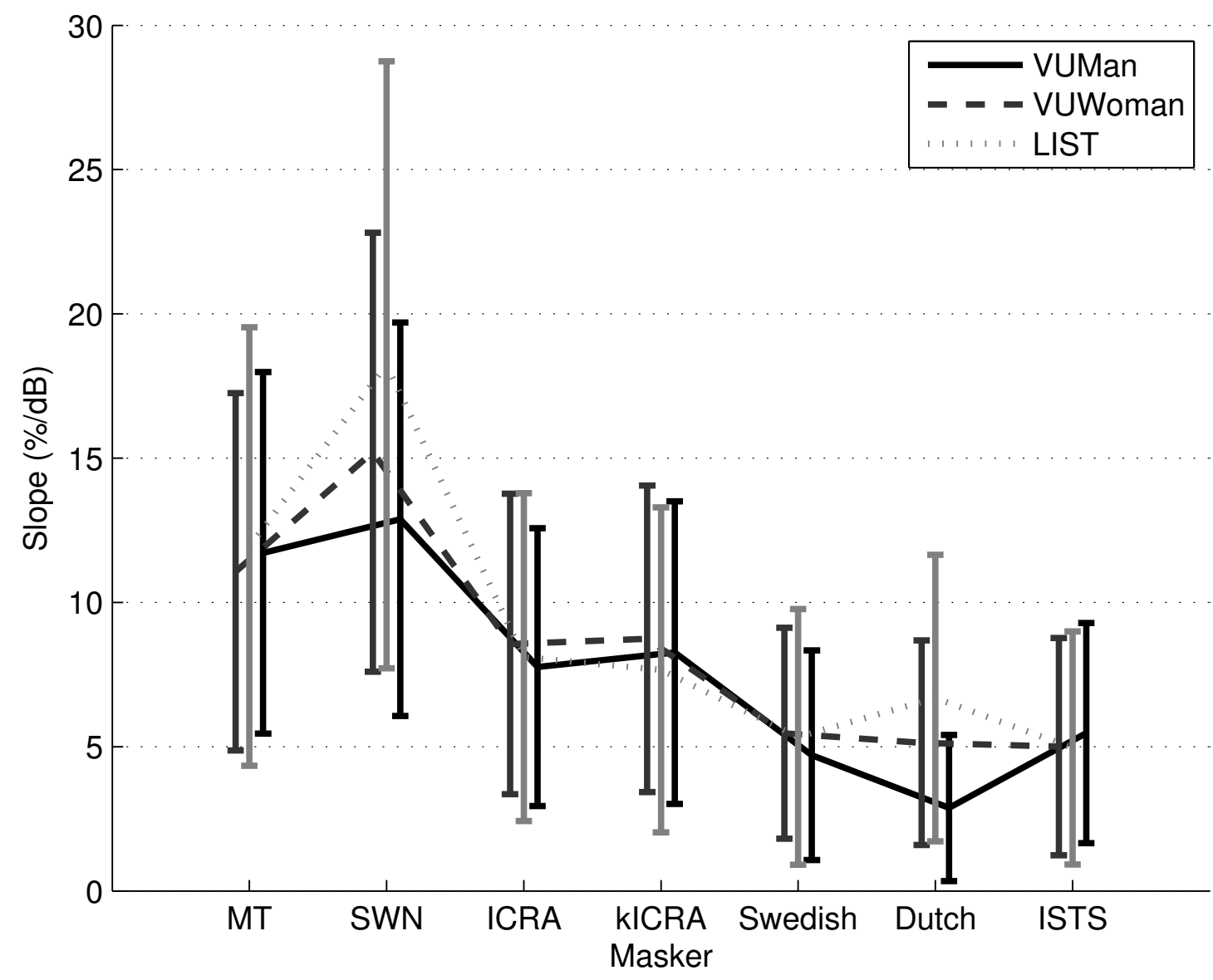

Figure 4: Slope of the psychometric function for the NH subjects, per speech material and masker. The error bars indicate $95 \%$ confidence intervals of the fit. 


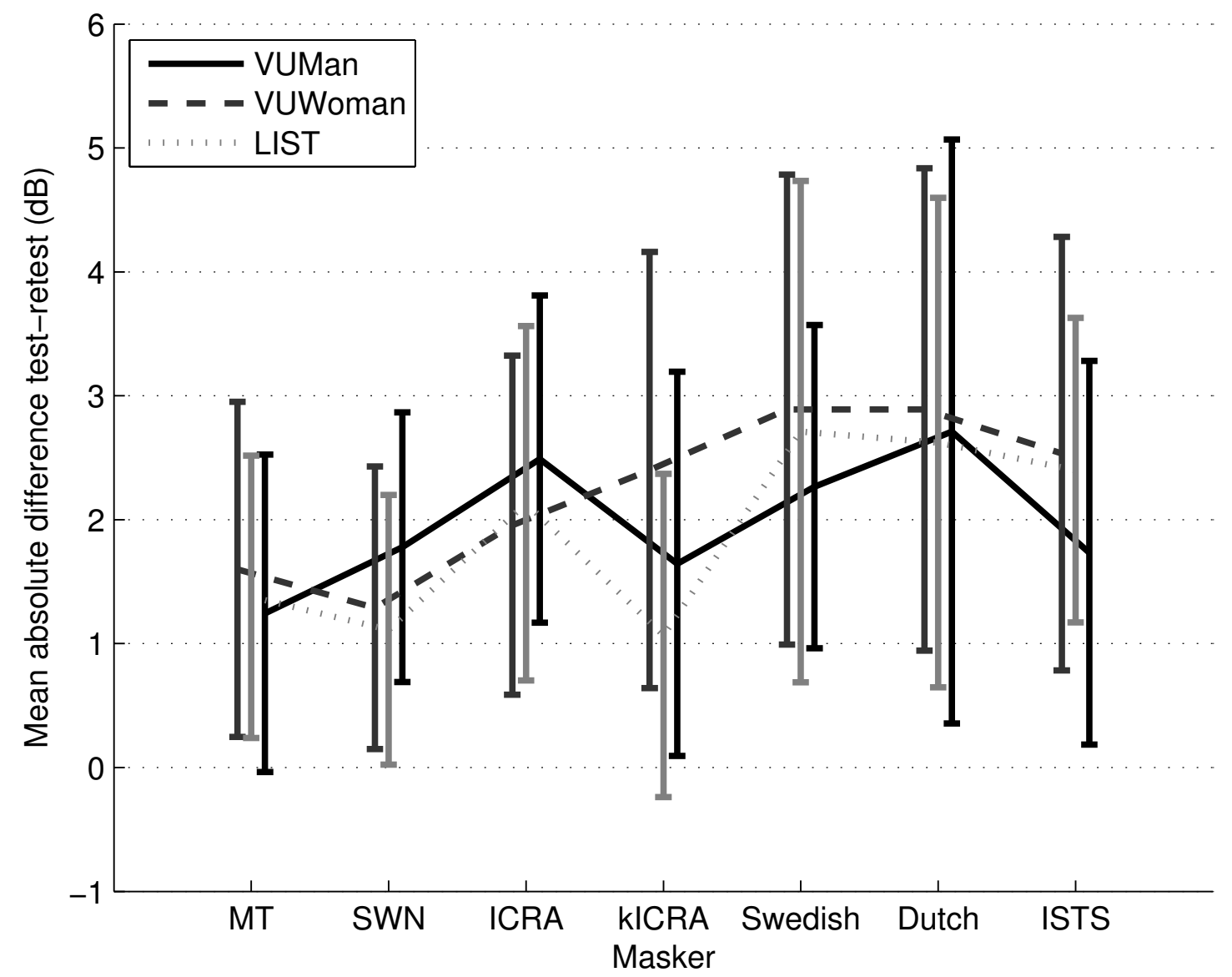

Figure 5: Mean absolute difference between test and retest for the NH subjects. Half the length of each error bar corresponds to the standard deviation on the mean. 


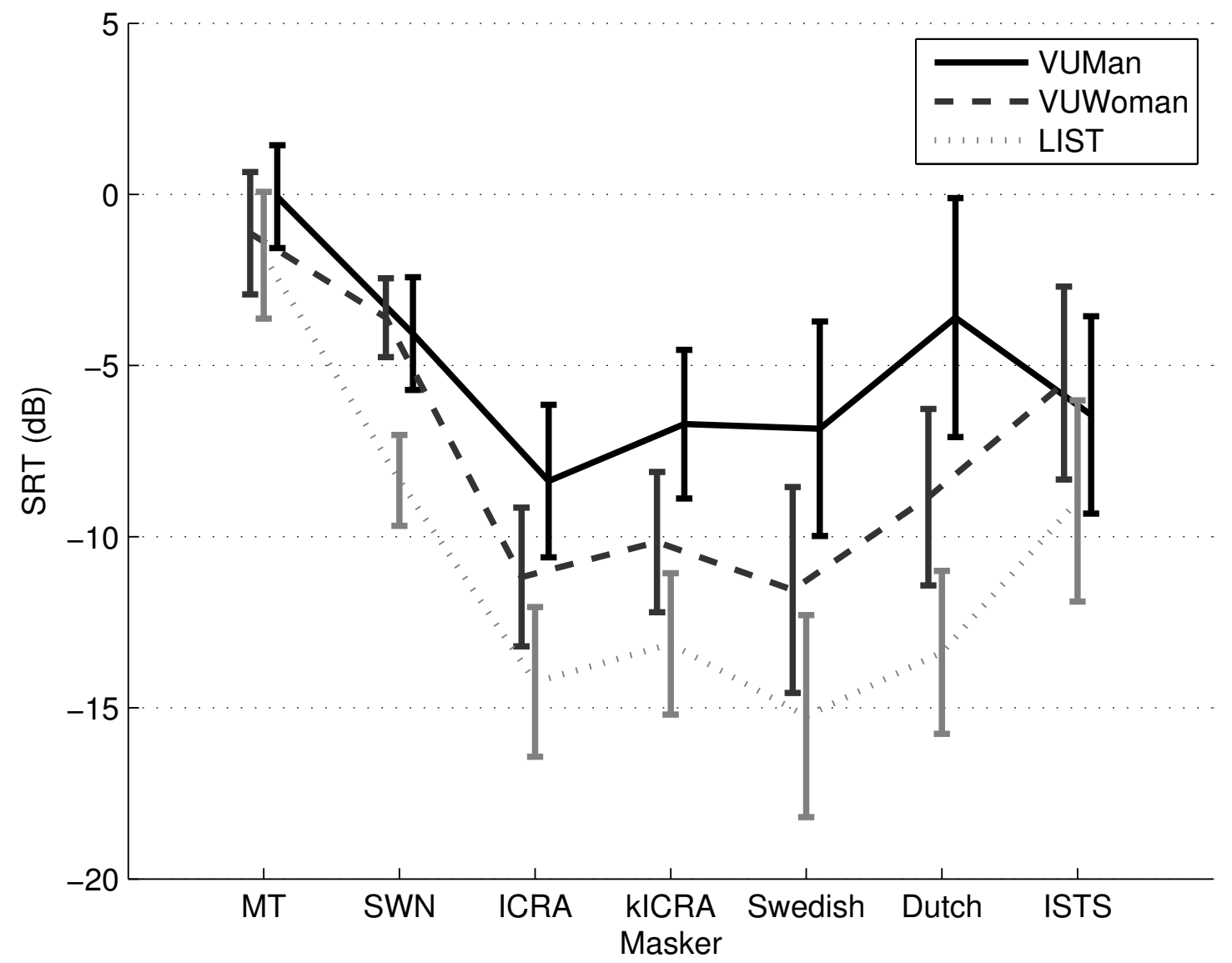

Figure 6: Mean SRTs for the NH subjects, grouped by speech material and masker. Half the length of an error bar corresponds to the standard deviation. 

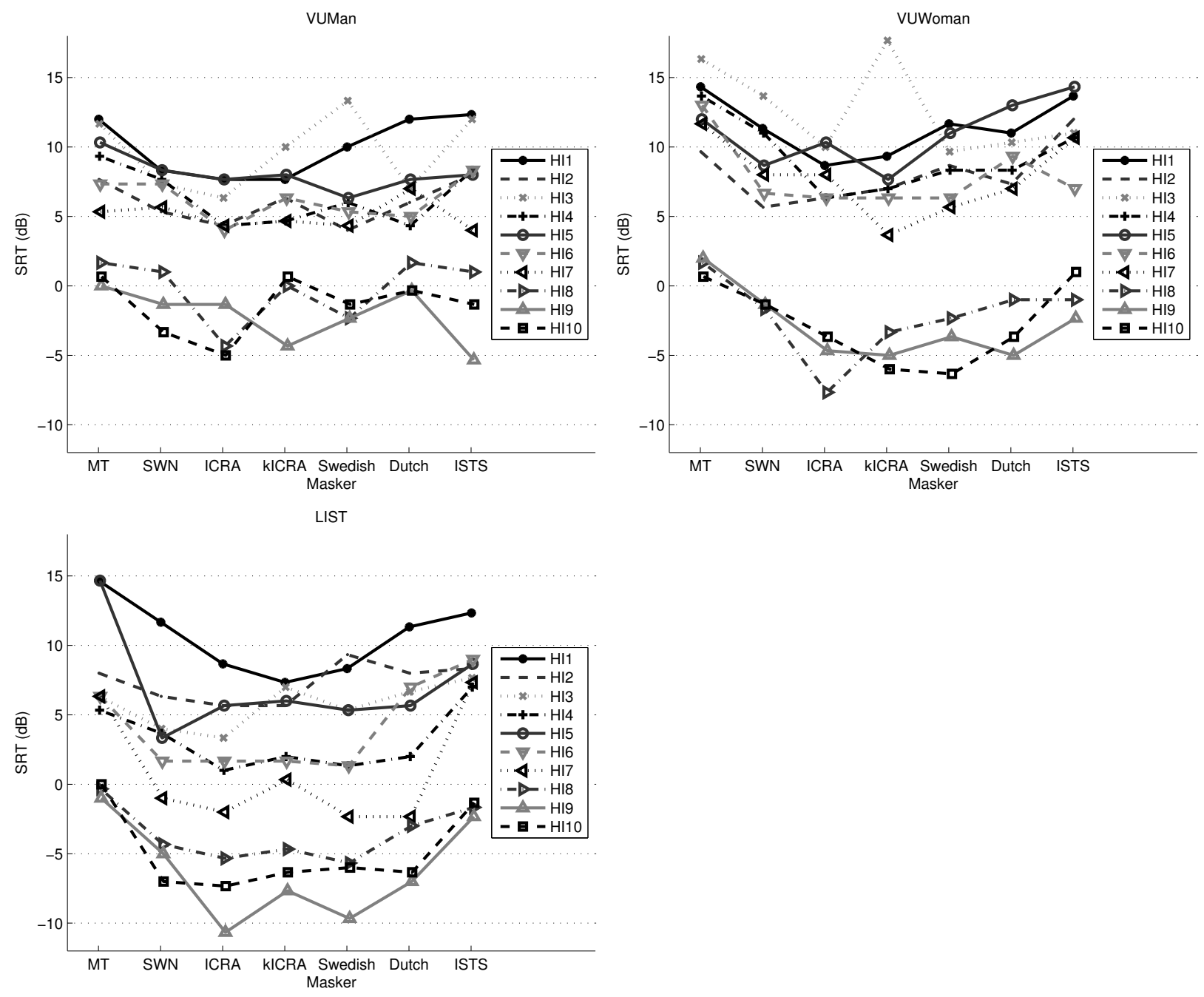

Figure 7: Mean (test-retest) SRTs for the HI subjects, grouped by speech material and masker. 


\section{$3.2 \quad$ SRT}

\subsubsection{Statistical analysis}

Speech recognition thresholds for the NH and HI subjects are shown in Figs. 6 and 7 and in Table IV. The NH and HI data were normally distributed, both across tests and across subjects, as verified with a Kolmogorov-Smirnov test. The NH data were analysed using a repeated measures ANOVA with variable SRT and factors speech material, masker and repetition (test/retest). There was no significant effect of repetition, indicating good test-retest reliability. Therefore, for each $\mathrm{NH}$ subject, test and retest data were used in group analyses. There was a main effect of speech material $(F(2,28)=242, p<0.01)$ and of masker $(F(6,28)=259, p<0.01)$ and of the interaction between speech material and masker $(F(12,28)=19, p<0.01)$. Comparisons between different levels of the factors were made using paired comparisons with Bonferroni correction on separate ANOVAs per speech material. In what follows, only p-values or significances will be given for the sake of brevity. A difference is considered significant if $p<0.05$.

The HI listeners are a heterogeneous group and their performance depends on individual hearing impairments and possible age-related factors. Therefore for this group only general tendencies will be considered.

\subsubsection{Norm values}

For the VU and LIST speech materials, norm values are available for the SRT obtained with NH subjects and the SWN masker. For the VUMan, VUWoman and LIST materials the respective norm values for the SRT with SWN (Cf. Table II) correspond well to the SRTs found for $10 \mathrm{NH}$ subjects in the current study (Cf. Table IV).

While the HI results cannot be compared to norm values, it should be noted that while audibility of the masker was verified for each subject and for each masker, the noise level of $60 \mathrm{dBA}$ may have been on the low side for HI1 and HI7. Care should be taken interpreting those results.

\subsubsection{Effect of masker type}

In what follows, SRTs will be compared between different maskers, for each of the three speech materials (VUMan, VUWoman and LIST). The maskers will be considered in order of increasing complexity, first adding TGs, then adding TFS and comprehensibility. First the steady-state maskers SWN and MT will be compared, followed by SWN (no TG, TFS or comprehensibility) and the fluctuating noises ICRA5-250 and kICRA (TG only). Thereafter kICRA will be compared to the Swedish competing talker and ISTS (TG and TFS) and to the Dutch competing talker (TG, TFS and comprehensibility). Generally, VUWoman displays the same tendencies as LIST (see Fig. 6). The differences in SRT between SWN (for which both materials were optimized) and each of the other maskers are nearly identical for these two materials.

While the SWN and MT maskers have similar properties (cf. Table III), performance for the $\mathrm{NH}$ listeners was significantly worse for the MT masker. For the VUMan, VUWoman and LIST respectively, the differences between SWN and MT are 4.0, 2.5, 6.6 dB (all $p<0.01$ ). The same tendency was seen for all HI listeners, except HI7 with VUMan. The average differences were 2.0, 3.4 and $4.7 \mathrm{~dB}$.

When TGs were present in the masker, performance improved significantly. For the VUMan, 
VUWoman and LIST respectively, an improvement in SRT of 4.3, 7.6 and $5.9 \mathrm{~dB}$ is seen between SWN and ICRA5-250 (all $p<0.01$ ). Comparing SWN and kICRA, improvements of 2.7, 6.6 and $4.8 \mathrm{~dB}$ were found (all $p<0.01$ ). The main difference between kICRA and ICRA5-250 is the maximal duration of the TGs (100 ms versus $250 \mathrm{~ms}$ ). Comparing kICRA an ICRA5-250, the SRT improved slightly by $1.8,1.0$ and $1.0 \mathrm{~dB}(p=0.03, p=0.15, p=0.06)$, for ICRA5-250. Visual inspection of the results for the HI subjects indicates a similar tendency for some subjects, but not for all of them and to a smaller extent. While for the $\mathrm{NH}$ listeners improvements of 4.3, 7.6 and $5.9 \mathrm{~dB}$ were found comparing SWN and ICRA5-250, for the HI listeners this was on average reduced to 1.8, 2.1 and $1.2 \mathrm{~dB}$ and for each individual $\mathrm{HI}$ listener the difference was smaller than the average difference for the NH listeners (except for one HI listener for VUMan).

The main difference between kICRA and Swedish or ISTS, is the availability of TFS information in the latter two maskers. Comparing kICRA and Swedish for the NH subjects, performance stayed the same for the VUMan and VUWoman but improved by $2.1 \mathrm{~dB}(p<0.01)$ for the LIST materials. Going from kICRA to ISTS, performance degraded significantly for the VUWoman and LIST by 4.6 and $4.2 \mathrm{~dB}$ (both $p<0.01$ ).

A comparison of Swedish and Dutch mainly indicates differences in performance caused by the comprehensibility of the masker. For the VUMan, VUWoman and LIST, performance decreased by $3.2,2.7$ and $1.9 \mathrm{~dB}$ from Swedish to Dutch $(p=0.18, p<0.01, p<0.01)$.

A comparison of the Swedish and ISTS maskers showed a significant decrease in performance for the ISTS masker of 6.0 and $6.3 \mathrm{~dB}$ for the VUWoman and LIST $(p<0.01)$ speech materials but no difference for the VUMan speech material.

\section{Discussion and conclusions}

In the next sections, the different masking factors will be analysed by dual comparisons between maskers. The idea is that estimates of the contribution of each factor to intelligibility can be estimated by a particular dual comparison. Then, assuming that this particular contribution remains constant for another situation, another dual comparison can be made to estimate the contribution of the next factor. In Fig. 8 and 9 the different effects are shown schematically. The influence of spectral differences between the maskers is taken into account by multiplying the difference in SII by $30 \mathrm{~dB}$ (ANSI-3.5 1997).

\subsection{Energetic masking}

Both NH and HI listeners scored worse for MT (20 competing talkers) than for SWN. In a similar comparison, Simpson \& Cooke (2005) found a significant difference between the condition with 16

competing talkers and speech-weighted noise. Based on the SII, differences in masker spectrum could account for approximately $3 \mathrm{~dB}$ of the difference (Cf. Fig. 3). A possible explanation for the remaining $1-3 \mathrm{~dB}$ difference is that, as opposed to the SWN, the MT signal contains frequent onsets (20 times more frequent than for a single talker), which distract the listener and increases forward masking (Simpson \& Cooke, 2005) .

Compared to SWN, performance with modulated maskers without TFS (kICRA and ICRA5-250) improved significantly for NH listeners, but not for HI listeners. This is consistent with previous studies (Festen \& Plomp, 1990; Gustafsson \& Arlinger, 1994; Peters et al., 1998; Wagener \& Brand, 2005; Wagener et al., 2006). SWN and kICRA differ mainly by the presence of TGs in the latter. The small differences between their spectra could account for 1.8/0.9/1.4 dB difference (for 


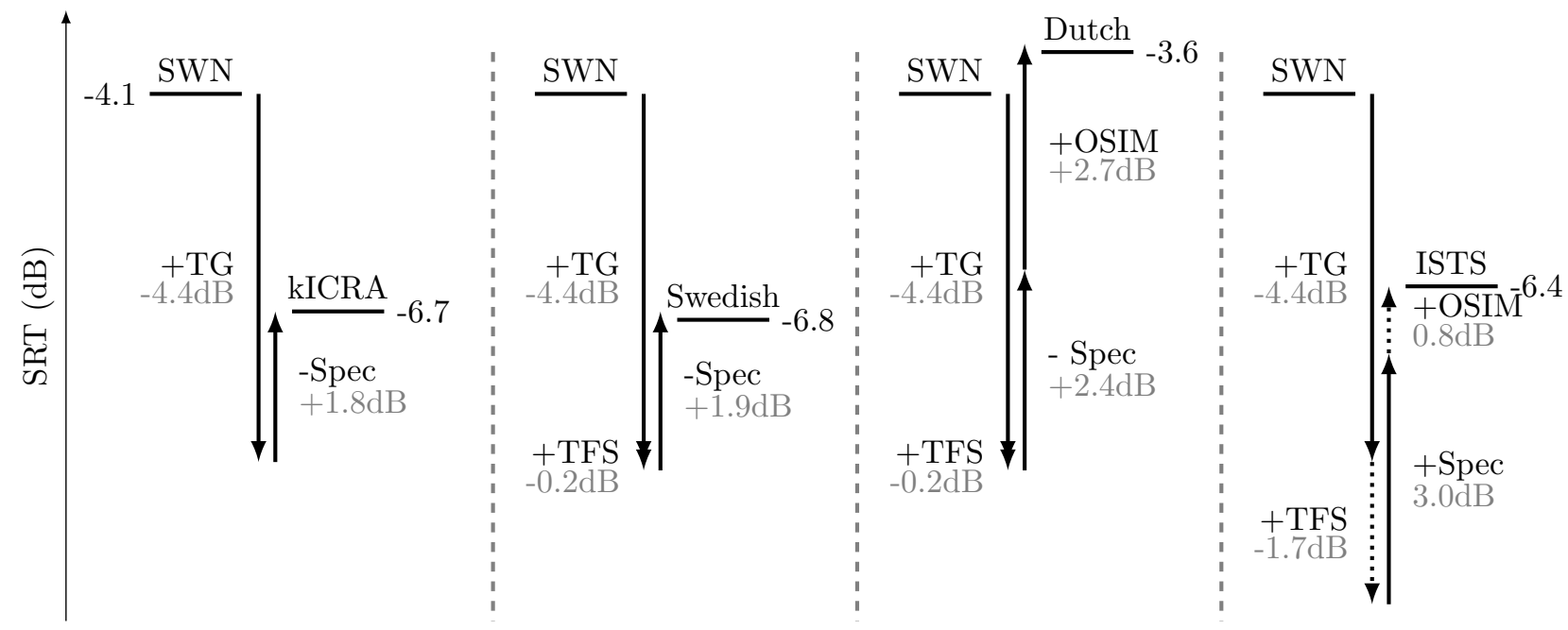

Figure 8: Factors influencing masking VUMan in NH listeners. +TG or + TFS indicates an improvement in performance due to the availability of TG or TFS in the masker. +OSIM indicates a decrease in performance due to increased object selection related informational masking. -Spec indicates a reduction in performance due to spectral differences.

VUMan, VUWoman and LIST respectively, Cf. Fig. 3 and the left panel of Figs. 8 and 9). Adding the SRT differences between SWN and kICRA $(2.6 / 6.6 / 4.8 \mathrm{~dB})$ to the estimated advantage due to spectral differences $(1.8 / 0.9 / 1.4 \mathrm{~dB})$, suggests than an improvement of about $4.4 / 7.5 / 6.1 \mathrm{~dB}$ was caused by listening in the gaps. It is currently unclear why the size of the effect differs so much between the different speech materials. The comparison between SWN and ICRA5-250 yielded similar results. For the NH subjects, performance was significantly better for the ICRA5-250 than for the kICRA for the VUMan (not shown in Fig. 8), and a similar tendency was present for VUWoman and LIST (not shown in Fig. 9). This was likely due to the difference in maximal gap length $(250 \mathrm{~ms}$ versus $100 \mathrm{~ms})$. Longer gaps give more opportunity to extract meaningful speech from them. However, if the gaps become too long, whole words might fall in a gap and decrease the test reproducibility. Figure 5 shows a tendency along those lines (comparing ICRA5-250 and kICRA).

\subsection{Informational masking}

When assessing the competing-talker conditions, IM comes into play. Three effects will be assessed: (1) the effect of OFIM when making TFS cues available (comparing kICRA with the competing talkers), (2) the effect of variation in the degree of OFIM based on similar or different TFS (comparing same and different-sex target and competing talkers), and (3) the OSIM caused by making the competing talker intelligible (comparing Swedish and Dutch). The different effects are shown schematically in Figs. 8 and 9. Comparison between different maskers in the NH subjects allows assessing the relative influence of energetic masking, OFIM and OSIM.

For the female talkers (VUWoman and LIST), the difference between kICRA and Swedish (1.3 and $2.0 \mathrm{~dB}$ ) indicates the gain due to the use of different TFS to separate the target and masker speakers (see panel 2 of Figs. 8 and 9). The difference was not significant for the VUWoman speech material. This could be due to the smaller difference in average $\mathrm{F}_{0}$ between the competing talker 


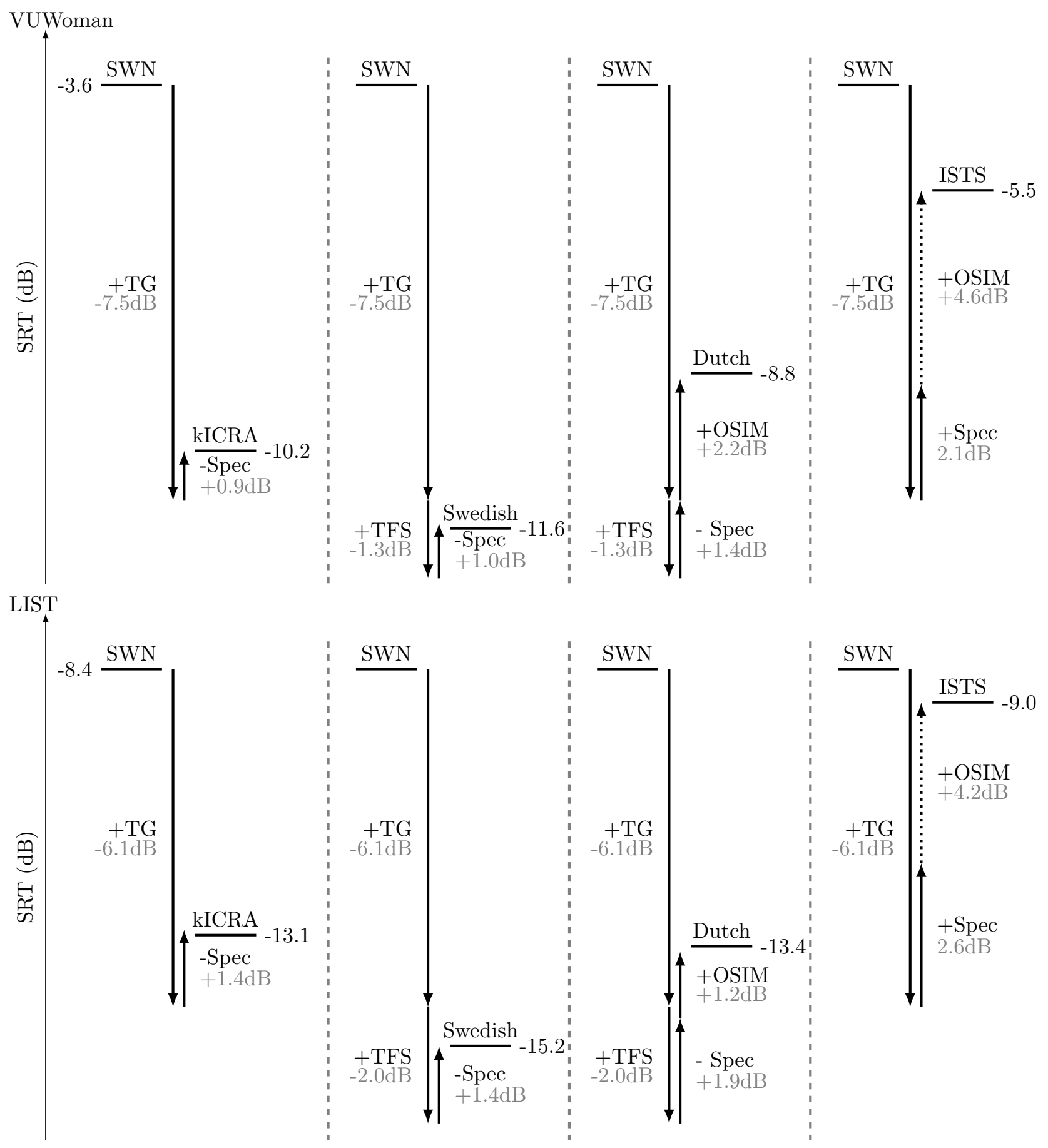

Figure 9: Factors influencing masking for VUWoman and LIST in NH listeners. +TG or + TFS indicates an improvement in performance due to the availability of TG or TFS in the masker. +OSIM indicates a decrease in performance due to increased object selection related informational masking. -Spec indicates a reduction in performance due to spectral differences. 
and VUWoman $(48 \mathrm{~Hz})$ than between the competing talker and LIST $(63 \mathrm{~Hz})$. For the VUMan, speech recognition performance did not improve, presumably because the TFS of the masker was very similar to the TFS of the target and thus provided no cue for object formation.

Comparison between Swedish and Dutch competing talkers shows the influence of comprehensibility of the masker (see panel three of Figs. 8 and 9). The Dutch masker provided 2.7, 2.2 and $1.2 \mathrm{~dB}$ more masking than the Swedish masker. This must be due to OSIM because the acoustic properties of the Swedish and Dutch masker are very similar. In a similar comparison, Rhebergen et al. (2005) estimated a difference of $\approx 6.6 \mathrm{~dB}$. However, they did not match the temporal properties of the maskers, as was done in the current study, and calculated this value from the results of several conditions, which could explain the difference in size of the effect.

Comparison between ISTS and kICRA with the female target speakers shows a large reduction in performance (see panel four of Figs. 8 and 9). If (1) release from masking by TGs is considered the same for ISTS and kICRA, (2) the influence of spectral differences is taken into account, and (3) there is little benefit of TFS because talker and masker are the same gender, there is a remaining difference of 4.6 and $4.2 \mathrm{~dB}$. This difference is presumably due to OSIM. This is confirmed by the fact that the subjects subjectively found the ISTS masker very distracting. The combination of languages sounded very unusual and listeners tended to try to determine the language of each segment. In the right panels of Figs. 8 and 9, the OSIM component is shown as a dotted line. For the VUMan there is an extra unknown component, i.e., the improvement in performance due to differing TFS cues (male target speaker and female masker). If we estimate this component as the average improvement found for the VUWoman and LIST materials $(1.7 \mathrm{~dB}$, comparing kICRA and Swedish maskers, the remaining OSIM component would be $0.8 \mathrm{~dB}$. This value is much smaller than the 4.6/4.2 dB found with the female target speakers. This can be due to our estimation of the effect of differing TFS or due to an additive effect of improved object formation, which reduces the bottom-up process that causes OSIM.

\subsection{General discussion and conclusions}

The magnitude of the effects of energetic and acoustic masking differed across speech materials. This indicates complicated interactions between the properties of the speech material and the masker (e.g., TFS, speech rate, voice timbre, etc.) and shows that norm values should be measured for each combination of speech material and masker.

While it is hard to draw strong conclusions from the HI data, due to the limited number of subjects and the heterogeneous nature of the group, they show similar tendencies as the NH data, but sometimes to a lesser extent, which may be related to individual degrees of hearing loss. Most importantly, the data seem to confirm that maskers containing TGs differentiate better between hearing impairments than steady state maskers, which is useful when evaluating patients' hearing. In future work, similar measurements with a much larger and more homogeneous HI population, combined with other tests such as measurement of psychophysical tuning curves, detection of dead regions and tests of cognitive function may shed more light on this issue.

The optimal masker type to be used depends on the purpose of the measurement. If measurement time is of primary concern, non-modulated maskers prevail, because of the steeper slopes of their psychometric functions. However, if assessing release of masking by the use of TGs, a masker with TGs can be considered. Such maskers have also been shown to differentiate better between degrees of hearing impairment (Festen \& Plomp, 1990; Gustafsson \& Arlinger, 1994; Peters et al., 1998; Wagener et al., 2006).

If perception of TFS is investigated or can have a substantial influence (e.g., in the evaluation of 
cochlear implant systems or of hearing aids that use TFS cues in their signal processing), a masker containing TFS should be used. Of the three maskers that were investigated in the current study, the Swedish competing talker seems the most promising: it does not have the large OSIM effect of the Dutch competing talker and it does not have the distracting effect of the ISTS masker. Of course, when using speech materials in languages other than Dutch, a language other than Swedish might be better suited for the foreign-language masker.

\section{Acknowledgements}

This research was carried out in the frame of the IWT (Institute for the Promotion of Innovation by Science and Technology in Flanders) project 080304 and was partly sponsored by Cochlear Ltd. We thank Jorien Loos for the collection and preliminary analysis of the data and our test subjects for their patient and enthusiastic participation in the experiments. We acknowledge Marcel Vlaming and Inga Holube for information about the ISTS. Koen Eneman was helpful in recording the Swedish and Dutch maskers. We thank Drs. Jacek Smurzynski, Stuart Rosen and two anonymous reviewers for their thorough reviews and helpful suggestions.

\section{References}

ANSI-3.5 1997, 1997. ANSI S3.5, methods for calculation of the speech intelligibility index. Acoustical Society of America.

Bacon S., Opie J. \& Montoya D., 1998. The Effects of Hearing Loss and Noise Masking on the Masking Release for Speech in Temporally Complex Backgrounds. J Speech Lang Hear Res, 41(3), $549-563$.

Bernstein J. \& Grant K., 2009. Auditory and auditory-visual intelligibility of speech in fluctuating maskers for normal-hearing and hearing-impaired listeners. J Acoust Soc Am, 125(5), 3358-72.

Boersma P. \& Weenink D., 2001. Praat, a system for doing phonetics by computer. Glot International, 5(9/10), 341-345.

Bregman A., 1990. Auditory scene analysis. MIT Press Cambridge, Mass.

Brungart D., 2001. Informational and energetic masking effects in the perception of two simultaneous talkers. J Acoust Soc Am, 109(3), 1101-9.

Brungart D., Chang P., Simpson B. \& Wang D., 2009. Multitalker speech perception with ideal time-frequency segregation: effects of voice characteristics and number of talkers. J Acoust Soc Am, 125(6), 4006-22.

Brungart D., Simpson B., Ericson M. \& Scott K., 2001. Informational and energetic masking effects in the perception of multiple simultaneous talkers. J Acoust Soc Am, 110(5 Pt 1), 2527-38.

Byrne D., Dillon H., Tran K., Arlinger S., Wilbraham K., Cox R., Hagerman B., Hetu R., Kei J., Lui C., Kiessling J., Kotby M., Nasser N., Kholy W., Nakanishi Y., Oyer H., Powell R., Stephens D., Meredith R., Sirimanna T., Tavartkiladze G., Frolenkov G., Westermann S. \& Ludvigsen C., 1994. An international comparison of long-term average speech spectra. J Acoust Soc Am, 96(4), $2108-2120$.

Cullington H. \& Zeng F., 2008. Speech recognition with varying numbers and types of competing talkers by normal-hearing, cochlear-implant, and implant simulation subjects. J Acoust Soc Am, 123(1), 450-61. 
Dreschler W., Verschuure H., Ludvigsen C. \& Westermann S., 2001. Icra noises: artificial noise signals with speech-like spectral and temporal properties for hearing instrument assessment. international collegium for rehabilitative audiology. Audiology, 40(3), 148-57.

Drullman R. \& Bronkhorst A., 2004. Speech perception and talker segregation: effects of level, pitch, and tactile support with multiple simultaneous talkers. J Acoust Soc Am, 116(5), 3090-8.

Durlach N., Mason C., Jr K., Arbogast T., Colburn H. \& Shinn-Cunningham B., 2003. Note on informational masking. $J$ Acoust Soc Am, 113(6), 2984-7.

Eisenberg L. \& Dirks D., 1995. Speech recognition in amplitude-modulated noise of listeners with normal and listeners with impaired hearing. J Speech Hear Res, 38(1), 222.

Festen J. \& Plomp R., 1990. Effects of fluctuating noise and interfering speech on the speechreception threshold for impaired and normal hearing. J Acoust Soc Am, 88(4), 1725-36.

Francart T., van Wieringen A. \& Wouters J., 2008. APEX 3: a multi-purpose test platform for auditory psychophysical experiments. J Neurosci Methods, 172(2), 283-293.

Gustafsson H. \& Arlinger S., 1994. Masking of speech by amplitude-modulated noise. J Acoust Soc Am, 95(1), 518-29.

Holube I., 2007. Short description of the international speech test signal (ists). Technical report, Hoertech.

Howard-Jones P. \& Rosen S., 1993. Uncomodulated glimpsing in "checkerboard" noise. J Acoust Soc Am, 93(5), 2915-22.

IPA, 1999, 1999. Handbook of the International Phonetic Association: a guide to the use of the International Phonetic Alphabet.

Kwon B. \& Turner C., 2001. Consonant identification under maskers with sinusoidal modulation: masking release or modulation interference? J Acoust Soc Am, 110(2), 1130-40.

Peters R., Moore B. \& Baer T., 1998. Speech reception thresholds in noise with and without spectral and temporal dips for hearing-impaired and normally hearing people. J Acoust Soc Am, 103(1), 577-87.

Plomp R. \& Mimpen A., 1979. Improving the reliability of testing the speech reception threshold for sentences. Audiology, 18(1), 43-52.

Rhebergen K., Versfeld N. \& Dreschler W., 2005. Release from informational masking by time reversal of native and non-native interfering speech. J Acoust Soc Am, 118(3 Pt 1), 1274-7.

Shinn-Cunningham B., 2008. Object-based auditory and visual attention. Trends Cogn Sci, 12(5), $182-6$.

Simpson S. \& Cooke M., 2005. Consonant identification in N-talker babble is a nonmonotonic function of N. J Acoust Soc Am, 118(5), 2775-8.

Takahashi G. \& Bacon S., 1992. Modulation Detection, Modulation Masking, and Speech Understanding in Noise in the Elderly. J Speech Hear Res, 35(6), 1410-1421.

van Wieringen A. \& Wouters J., 2008. LIST and LINT: sentences and numbers for quantifying speech understanding in severely impaired listeners for Flanders and The Netherlands. Int $J$ Audol, $47(6), 348-355$.

Versfeld N., Daalder L., Festen J. \& Houtgast T., 2000. Method for the selection of sentence materials for efficient measurement of the speech reception threshold. J Acoust Soc Am, 107(3), 1671-84. 
Wagener K. \& Brand T., 2005. Sentence intelligibility in noise for listeners with normal hearing and hearing impairment: influence of measurement procedure and masking parameters. Int $J$ Audiol, 44(3), 144-56.

Wagener K., Brand T. \& Kollmeier B., 2006. The role of silent intervals for sentence intelligibility in fluctuating noise in hearing-impaired listeners. Int J Audiol, 45(1), 26-33. 


\begin{tabular}{rccccl}
\hline Subject & Sex & Age (years) & PTA (dB HL) & Test ear & Degree \\
\hline HI1 & $\uparrow$ & 65 & 60 & $\mathrm{R}$ & moderate-severe \\
HI2 & $\wp$ & 77 & 46 & $\mathrm{R}$ & moderate \\
HI3 & $\wp$ & 53 & 43 & $\mathrm{~L}$ & moderate \\
HI4 & $0^{x}$ & 51 & 53 & $\mathrm{R}$ & moderate \\
HI5 & $0^{x}$ & 74 & 30 & $\mathrm{R}$ & mild \\
HI6 & $0^{x}$ & 68 & 33 & $\mathrm{~L}$ & mild \\
HI7 & $0^{x}$ & 78 & 40 & $\mathrm{~L}$ & moderate \\
HI8 & $\uparrow$ & 20 & 42 & $\mathrm{R}$ & moderate \\
HI9 & $0^{x}$ & 57 & 10 & $\mathrm{R}$ & mild \\
HI10 & $\uparrow$ & 46 & 36 & $\mathrm{R}$ & mild \\
\hline
\end{tabular}

Table I: Overview of HI subjects. PTA is the Pure-Tone Average (average hearing threshold at 500,1000 and $2000 \mathrm{~Hz}$ of the tested ear). 


\begin{tabular}{rcccc}
\hline Speech material & Sex & $\mathrm{F}_{0}(\mathrm{~Hz})$ & Speed $(\mathrm{syl} / \mathrm{s})$ & Norm SRT $(\mathrm{dB}$ SNR) \\
\hline VUMan & $\sigma^{7}$ & $113 \pm 28$ & 4.7 & $-4.0 \pm 0.8$ \\
VUWoman & + & $179 \pm 47$ & 4.7 & $-4.1 \pm 0.8$ \\
LIST & $\subsetneq$ & $194 \pm 34$ & 2.5 & $-8.0 \pm 0.2$ \\
\hline
\end{tabular}

Table II: Speech material characteristics. $\mathrm{F}_{0}$ is the average pitch, calculated using Praat (Boersma \& Weenink, 2001). Norm SRTs are given for the SWN corresponding to the speech material (van Wieringen \& Wouters, 2008; Versfeld et al., 2000). 


\begin{tabular}{rccccccc}
\hline Masker & F $_{0}$ & Sex & Spectrum & Speed (syl/s) & TG & TFS & Comprehensible \\
\hline SWN & N/A & N/A & $\sigma^{7} / q$ & N/A & & & \\
MT & N/A & N/A & $\sigma^{7} \& q$ & N/A & & $\checkmark$ & \\
ICRA5-250 & N/A & N/A & $\sigma^{7}$ & $?$ & $\checkmark$ & & \\
kICRA & N/A & N/A & $\sigma^{7}$ & 5.6 & $\checkmark$ & & \\
Swedish & $131 \pm 40$ & $\sigma^{7}$ & $\sigma^{7}$ & 4.2 & $\checkmark$ & $\checkmark$ & \\
Dutch & $126 \pm 51$ & $\sigma^{7}$ & $\sigma^{7}$ & 5.6 & $\checkmark$ & $\checkmark$ & $\checkmark$ \\
ISTS & $198 \pm 44$ & $q$ & $q$ & 5.6 & $\checkmark$ & $\checkmark$ & \\
\hline
\end{tabular}

Table III: Overview of masker properties. $\mathrm{F}_{0}$ is the average fundamental frequency, in Hz. "Sex" is the sex of the speaker (Male or Female). "TG" indicates the presence of temporal gaps. "TFS" indicates the presence of temporal fine structure. "Comprehensible" refers to the extent to which the masker is understood by the subjects. 


\begin{tabular}{|c|c|c|c|c|c|c|c|}
\hline & MT & SWN & ICRA & kICRA & Swedish & Dutch & ISTS \\
\hline VUMan & $-0.1 \pm 1.5$ & $-4.1 \pm 1.6$ & $-8.4 \pm 2.2$ & $-6.7 \pm 2.2$ & $-6.8 \pm 3.1$ & $-3.6 \pm 3.5$ & $-6.4 \pm 2.9$ \\
\hline VUWoman & $-1.1 \pm 1.8$ & $-3.6 \pm 1.2$ & $-11.2 \pm 2.0$ & $-10.2 \pm 2.0$ & $-11.6 \pm 3.0$ & $-8.8 \pm 2.6$ & $-5.5 \pm 2.8$ \\
\hline LIST & $-1.8 \pm 1.9$ & $-8.4 \pm 1.3$ & $-14.2 \pm 2.2$ & $-13.1 \pm 2.1$ & $-15.2 \pm 3.0$ & $-13.4 \pm 2.4$ & $-9.0 \pm 2.9$ \\
\hline
\end{tabular}

Table IV: SRTs \pm 1 standard deviation in $\mathrm{dB}$ SNR for NH subjects 


\section{List of Tables}

I Overview of HI subjects. PTA is the Pure-Tone Average (average hearing threshold at 500, 1000 and $2000 \mathrm{~Hz}$ of the tested ear). . . . . . . . . . . . . . . . 24

II Speech material characteristics. $F_{0}$ is the average pitch, calculated using Praat (Boersma \& Weenink, 2001). Norm SRTs are given for the SWN corresponding to the speech material (van Wieringen \& Wouters, 2008; Versfeld et al., 2000). . . . .

III Overview of masker properties. $\mathrm{F}_{0}$ is the average fundamental frequency, in $\mathrm{Hz}$. "Sex" is the sex of the speaker (Male or Female). "TG" indicates the presence of temporal gaps. "TFS" indicates the presence of temporal fine structure. "Comprehensible" refers to the extent to which the masker is understood by the subjects. . . . . . . . 26

IV SRTs \pm 1 standard deviation in dB SNR for NH subjects . . . . . . . . . . . . . 27 


\section{List of Figures}

1 Average audiogram of the NH subjects and individual unaided audiograms for the HI subjects. . . . . . . . . . . . . . . . . . . . .

2 Extract of the masker envelopes, determined by full wave rectification and low pass filtering $\left(\mathrm{f}_{\mathrm{c}}=25 \mathrm{~Hz}\right)$ of an extract of the masker. All input signals were normalized to the same RMS level. . . . . . . . . . . . . . . . . . . . . .

3 SII at $0 \mathrm{~dB}$ SNR for each combination of speech material and masker. The axis on the right hand side indicates that a difference in SII of 0.1 corresponds to a

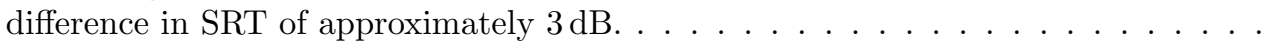

4 Slope of the psychometric function for the NH subjects, per speech material and masker. The error bars indicate $95 \%$ confidence intervals of the fit. . . . . . . . .

5 Mean absolute difference between test and retest for the NH subjects. Half the length of each error bar corresponds to the standard deviation on the mean. . . . .

6 Mean SRTs for the NH subjects, grouped by speech material and masker. Half the length of an error bar corresponds to the standard deviation. . . . . . . . . . . .

7 Mean (test-retest) SRTs for the HI subjects, grouped by speech material and masker. . . . . . . . . . . . . . . . . . . . .

8 Factors influencing masking VUMan in NH listeners. +TG or + TFS indicates an improvement in performance due to the availability of TG or TFS in the masker. +OSIM indicates a decrease in performance due to increased object selection related informational masking. -Spec indicates a reduction in performance due to spectral

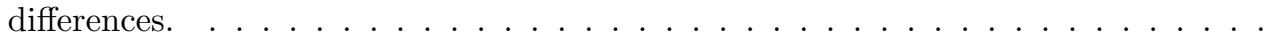

9 Factors influencing masking for VUWoman and LIST in NH listeners. +TG or +TFS indicates an improvement in performance due to the availability of TG or TFS in the masker. +OSIM indicates a decrease in performance due to increased object selection related informational masking. -Spec indicates a reduction in performance due to spectral differences. . . . . . . . . . . . . . . . . 